\title{
Ultrathin Trimetal-Organic Framework Nanosheet Electrocatalysts for the Highly Efficient Oxygen Evolution Reaction
}

Songyang Niu, ${ }^{1}$ Chenhui Li, ${ }^{1}$ Jia Huo, ${ }^{1,2, ~ *}$ Wanrong Dong ${ }^{1,}$ * Samir El Hankari, ${ }^{1,3,}{ }^{3} Y u$ Liang, ${ }^{1}$ Qiling $\mathrm{Li}^{1}$

${ }^{1}$ State Key Laboratory of Chem/Bio-Sensing and Chemometrics, Provincial Hunan Key Laboratory for Graphene Materials and Devices, College of Chemistry and Chemical Engineering, Hunan University, Changsha, 410082, Hunan, China

${ }^{2}$ Hunan Provincial Key Laboratory of Advanced Materials for New Energy Storage and Conversion, Hunan University of Science and Technology, Xiangtan 411201, China

${ }^{3}$ Department of Chemistry, Mohammed VI Polytechnic University, Ben Guerir 43150, Morocco

*To whom correspondence should be addressed: E-mail: jiahuo@hnu.edu.cn; wanrongdong@hnu.edu.cn; elhankarisamir@gmail.com 
Results and Discussion

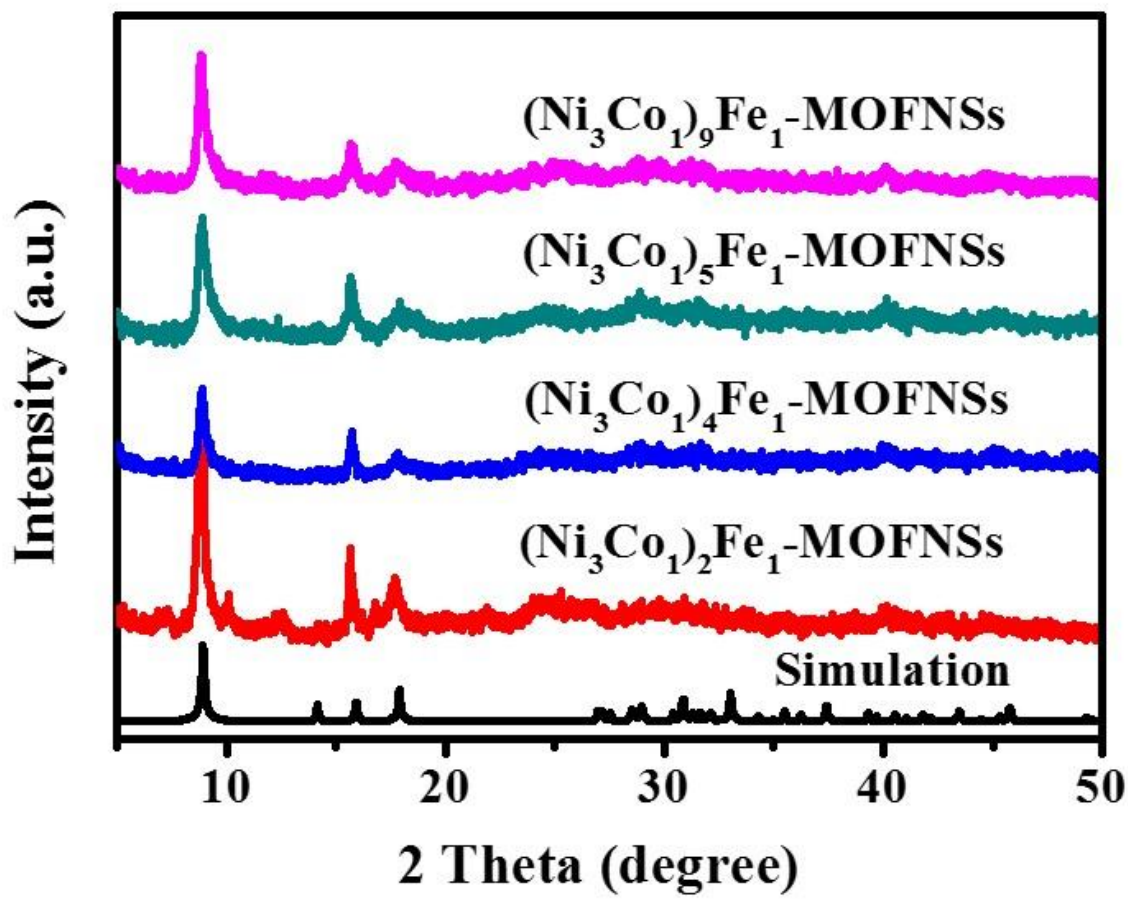

Figure S1 The powder X-ray diffraction (PXRD) patterns of different metal ratio of NiCoFe-MOFNSs

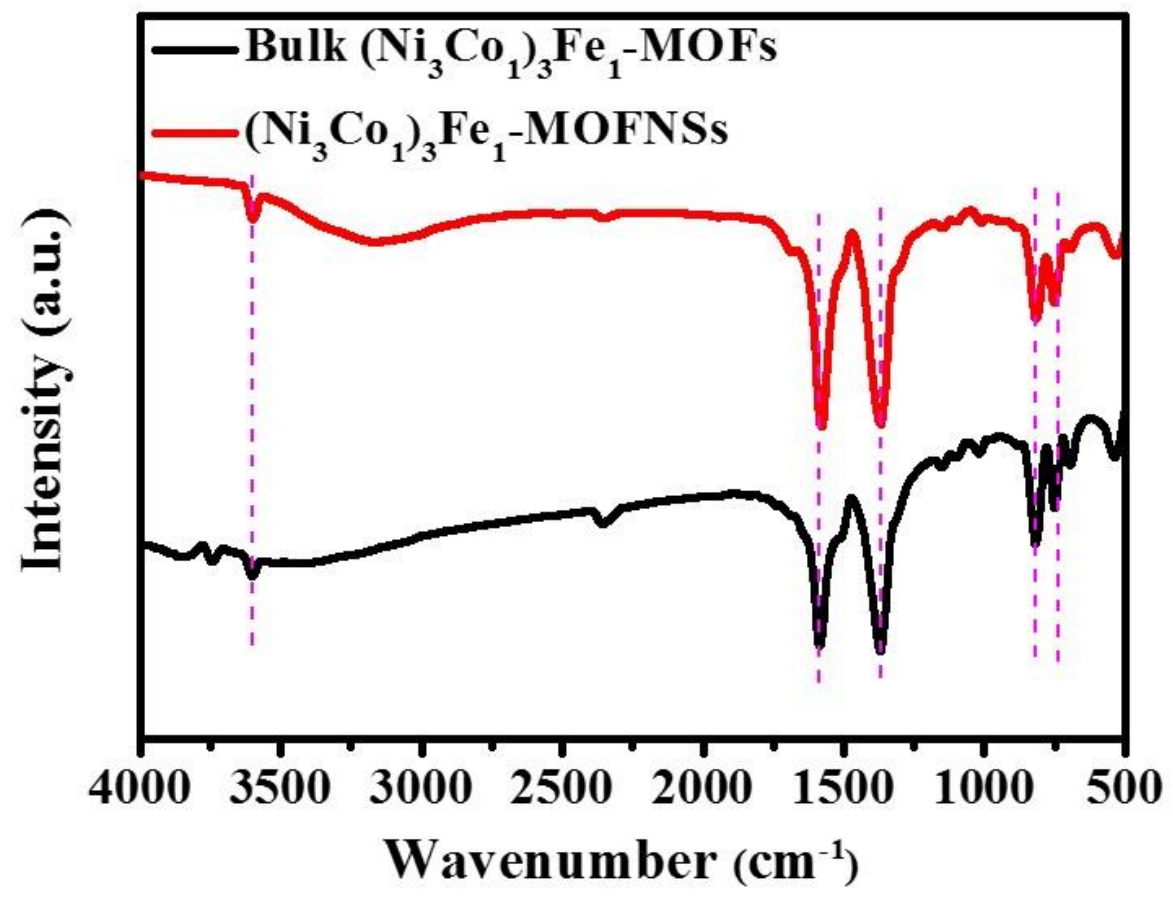

Figure S2 FT-IR spectra of $\left(\mathrm{Ni}_{3} \mathrm{Co}_{1}\right)_{3} \mathrm{Fe}_{1}-\mathrm{MOFNSs}$ and bulk $\left(\mathrm{Ni}_{3} \mathrm{Co}_{1}\right)_{3} \mathrm{Fe}_{1}-\mathrm{MOFs}$. 

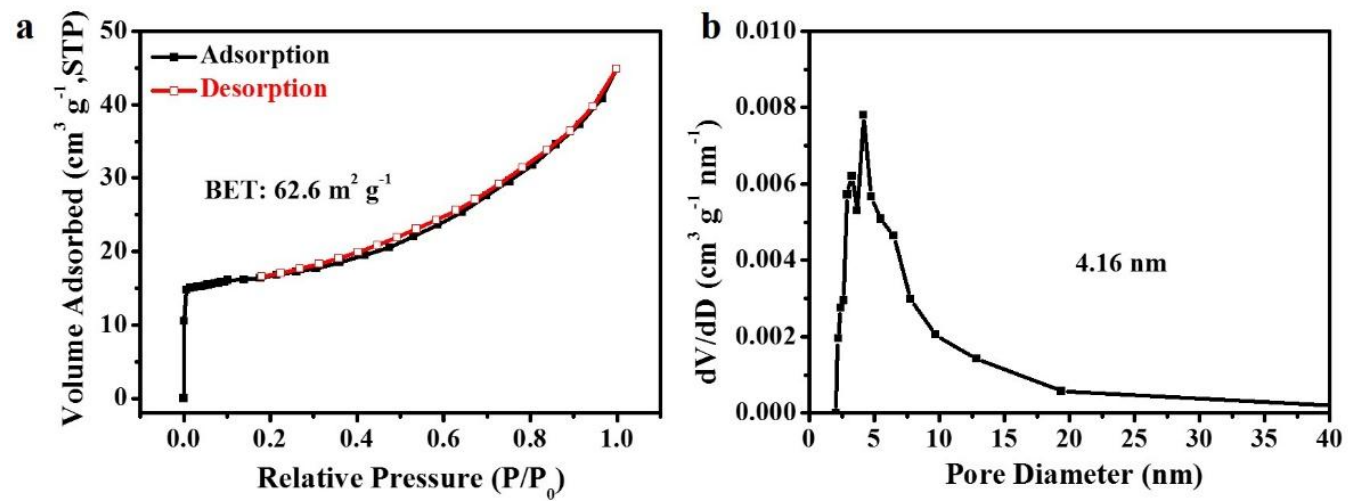

Figure $\mathbf{S 3} \mathrm{N}_{2}$ sorption isotherm (a) and the corresponding average pore size and pore size distributions (b) derived from the adsorption branch by using Barrett-JoyerHalenda (BJH) method.
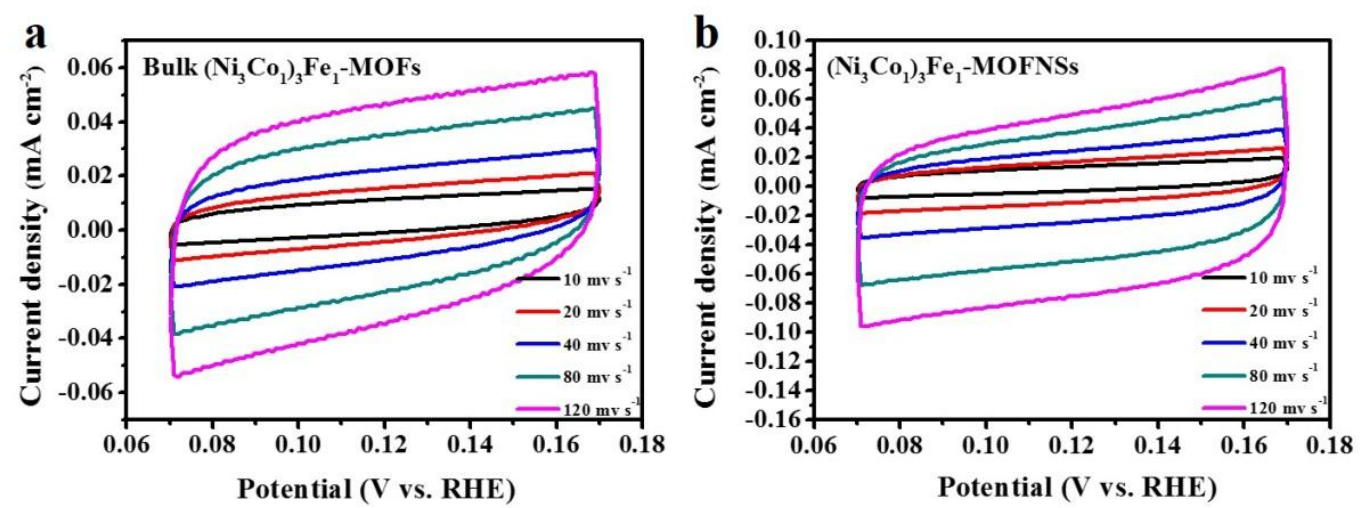

Figure S4 CV curves at different scan rates of bulk $\left(\mathrm{Ni}_{3} \mathrm{Co}_{1}\right)_{3} \mathrm{Fe}_{1}-\mathrm{MOF}$ (a) and $\left(\mathrm{Ni}_{3} \mathrm{Co}_{1}\right)_{3} \mathrm{Fe}_{1}$-MOFNSs (b). 

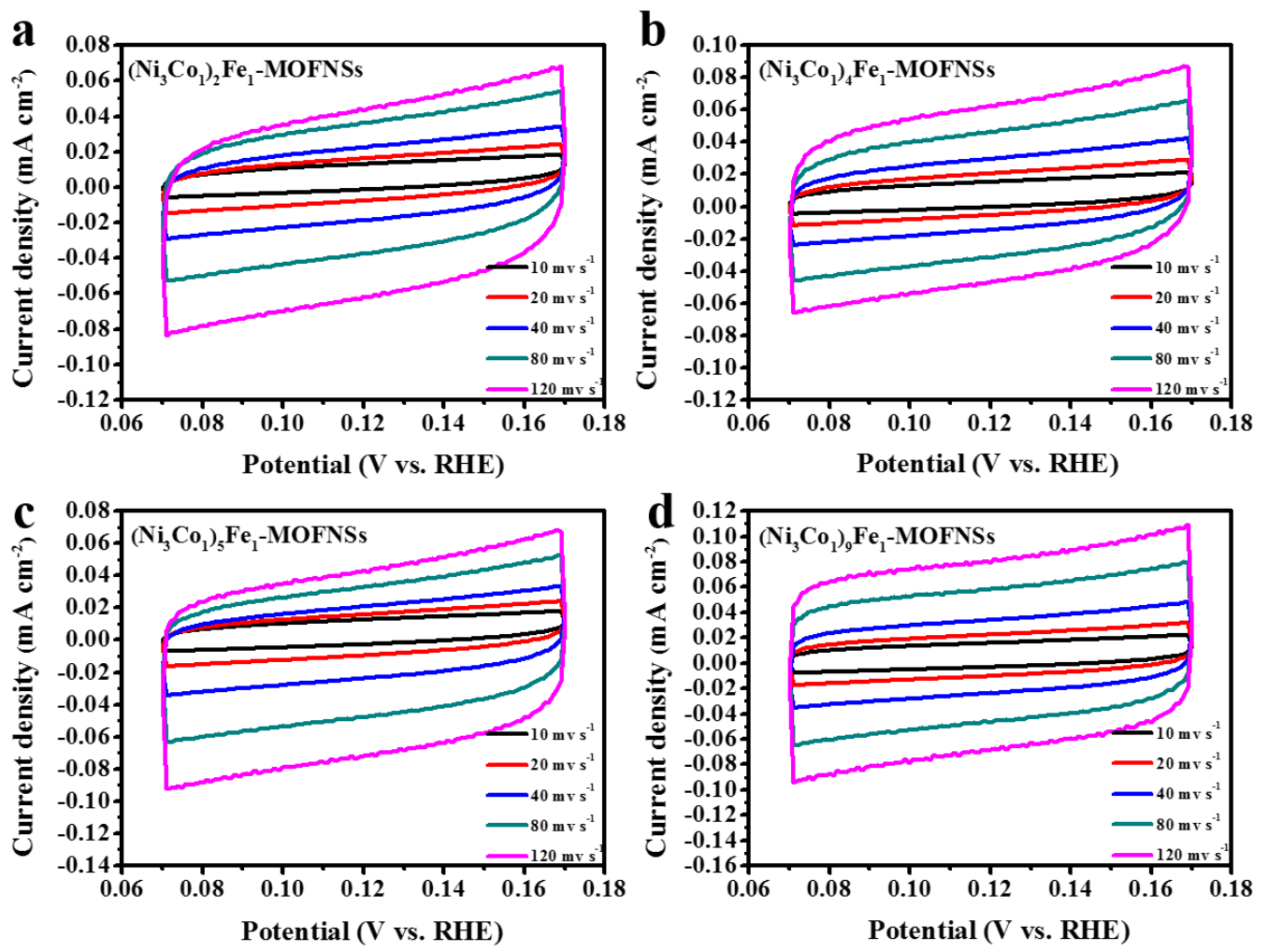

Figure S5 CV curves at different scan rates of different metal ratio (a-d) of NiCoFe-MOFNSs.

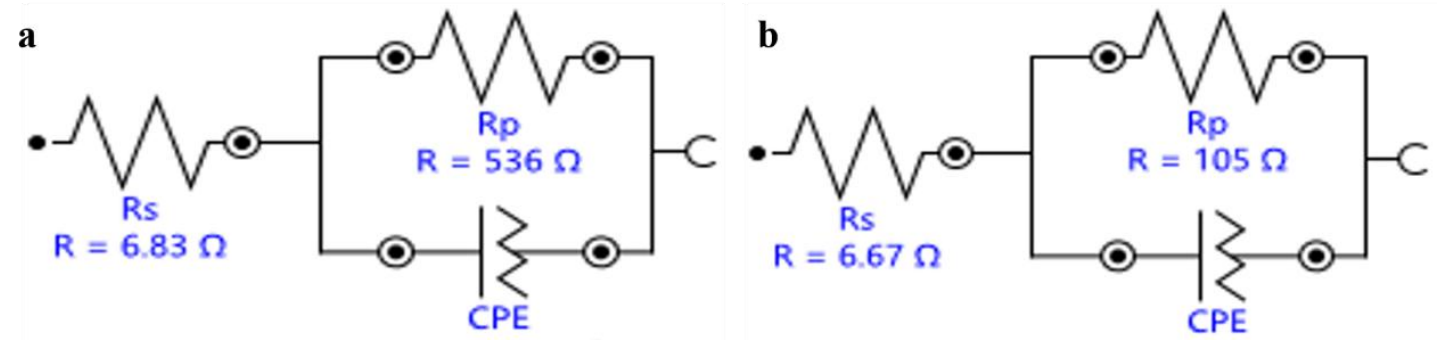

Figure S6 The electrochemical impedance spectroscopy (EIS) in $1.0 \mathrm{~m} \mathrm{KOH}$ solution at an applied potential of $1.45 \mathrm{~V}$ (vs RHE). (a) Bulk $\left(\mathrm{Ni}_{3} \mathrm{Co}_{1}\right)_{3} \mathrm{Fe}_{1}$-MOFs; (b) $\left(\mathrm{Ni}_{3} \mathrm{Co}_{1}\right)_{3} \mathrm{Fe}_{1}-\mathrm{MOFNSs}$. Rs: electrolyte resistance, Rp: charge-transfer resistance, CPE: constant-phase element. 


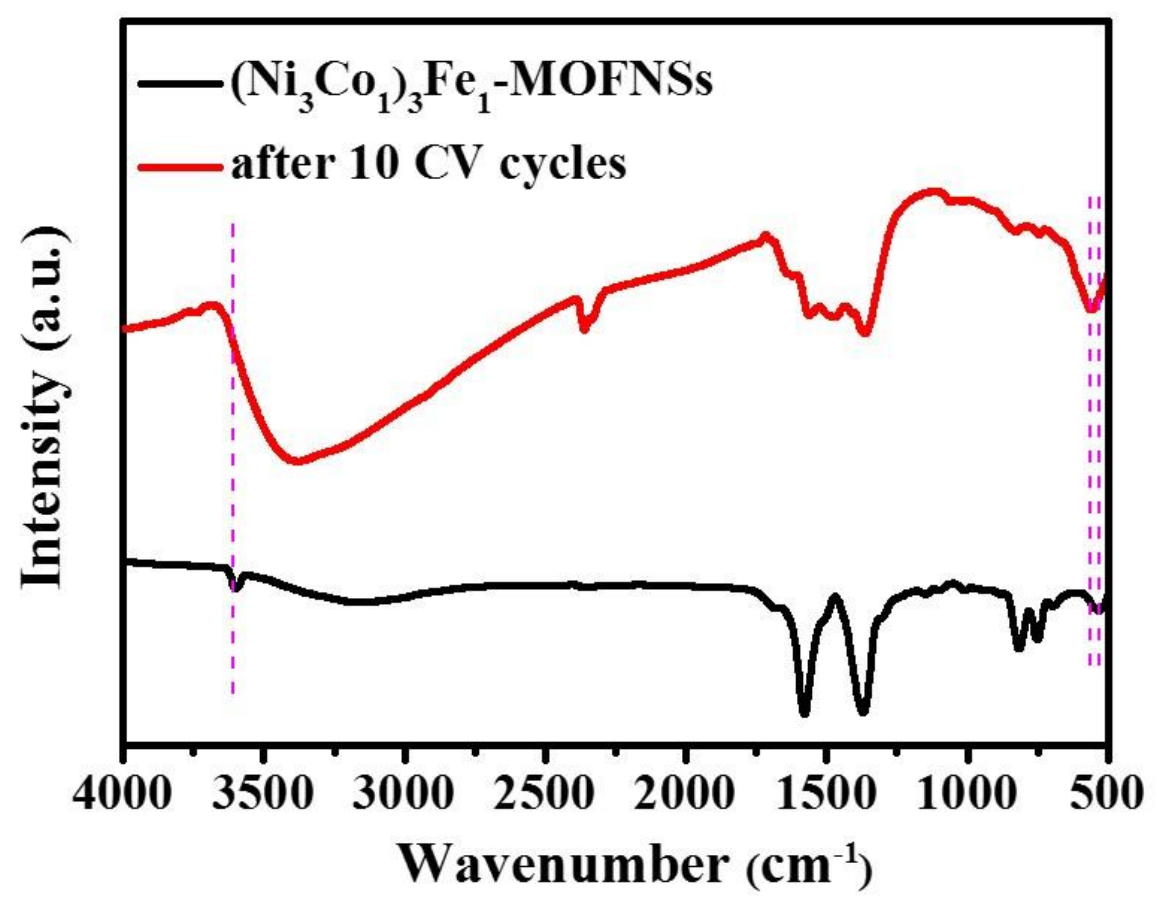

Figure S7 FT-IR spectra of $\left(\mathrm{Ni}_{3} \mathrm{Co}_{1}\right)_{3} \mathrm{Fe}_{1}$-MOFNSs and after $10 \mathrm{CV}$ cycles.
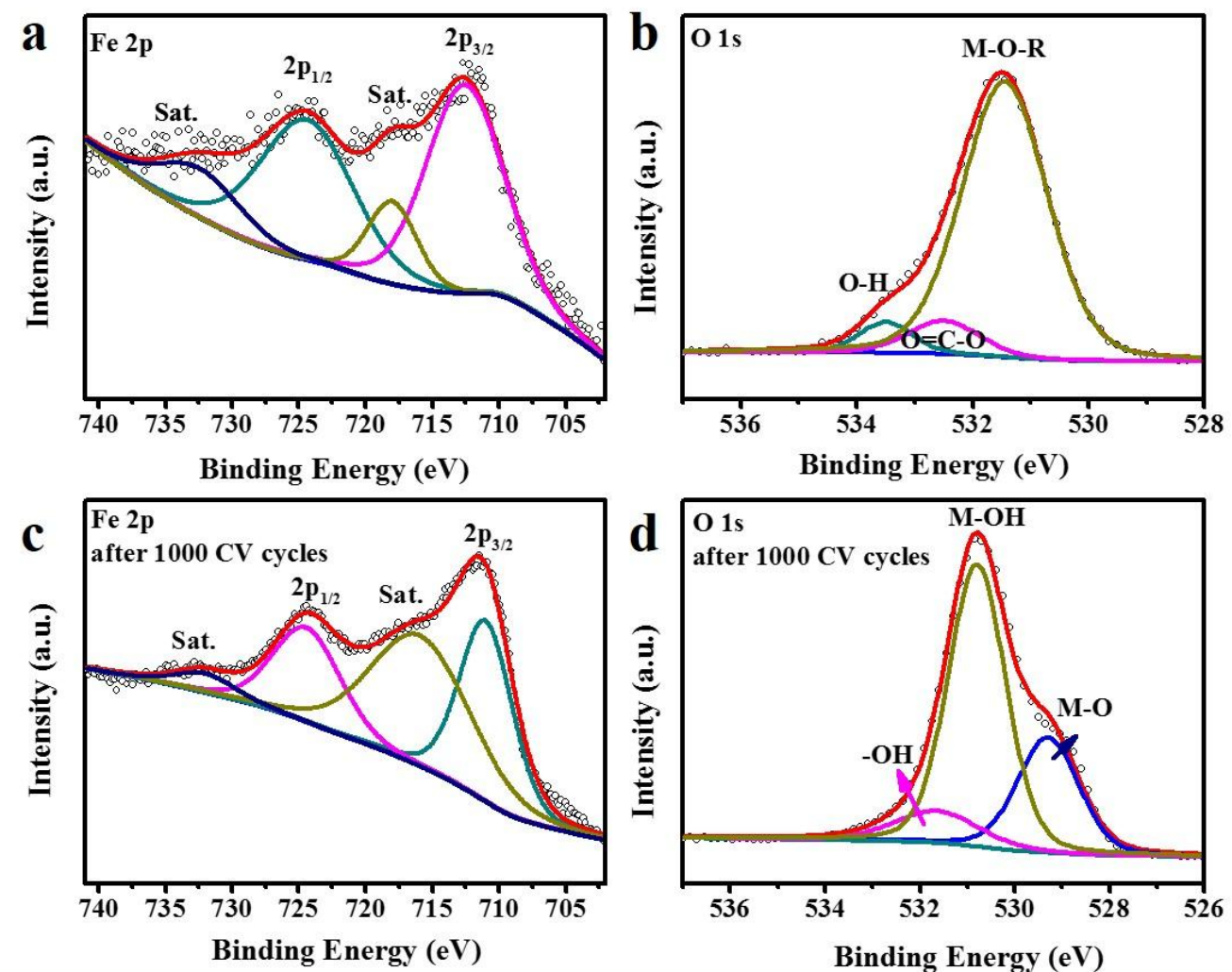

Figure S8 (a), (b) (c) and (d) $\mathrm{Fe} 2 \mathrm{p}$ and $\mathrm{O}$ 1s XPS of $\left(\mathrm{Ni}_{3} \mathrm{Co}_{1}\right)_{3} \mathrm{Fe}_{1}-\mathrm{MOFNSs}$ before and after $1000 \mathrm{CV}$ cycles. 
Table S1. Comparisons of OER performance for various MOF-based electrocatalysts.

\begin{tabular}{|c|c|c|c|c|}
\hline Catalyst & Substrate & Electrolyte & $\eta(\mathrm{mV})$ & Reference \\
\hline $\begin{array}{c}\left(\mathrm{Ni}_{3} \mathrm{Co}_{1}\right)_{1} \mathrm{Fe}_{1}-\mathrm{MOF} \\
\mathrm{NSs}\end{array}$ & GCE & $1.0 \mathrm{M} \mathrm{KOH}$ & $245 @ 10 \mathrm{~mA} \cdot \mathrm{cm}^{-2}$ & This work \\
\hline NiCo-UMOFNs & GCE & $1.0 \mathrm{M} \mathrm{KOH}$ & $250 @ 10 \mathrm{~mA} \cdot \mathrm{cm}^{-2}$ & 1 \\
\hline Ni-MOF@Fe-MOF & GCE & $1.0 \mathrm{M} \mathrm{KOH}$ & $265 @ 10 \mathrm{~mA} \cdot \mathrm{cm}^{-2}$ & 2 \\
\hline NiFe-UMNs & GCE & $1.0 \mathrm{M} \mathrm{KOH}$ & $260 @ 10 \mathrm{~mA} \cdot \mathrm{cm}^{-2}$ & 3 \\
\hline $\begin{array}{c}\left(\mathrm{Ni}_{2} \mathrm{Co}_{1}\right)_{0.925} \mathrm{Fe}_{0.075^{-}} \\
\text {MOF-NF }\end{array}$ & GCE & $1.0 \mathrm{M} \mathrm{KOH}$ & $257 @ 10 \mathrm{~mA} \cdot \mathrm{cm}^{-2}$ & 4 \\
\hline NiCoFe-MOF & GCE & $1.0 \mathrm{M} \mathrm{KOH}$ & $320 @ 10 \mathrm{~mA} \cdot \mathrm{cm}^{-2}$ & 5 \\
\hline $\begin{array}{c}\text { 2D Co-MOF } \\
\text { nanosheet }\end{array}$ & GCE & $1.0 \mathrm{M} \mathrm{KOH}$ & $263 @ 10 \mathrm{~mA} \cdot \mathrm{cm}^{-2}$ & 6 \\
\hline
\end{tabular}

\section{References}

(1) Zhao, S.; Wang, Y.; Dong, J.; He, C.-T.; Yin, H.; An, P.; Zhao, K.; Zhang, X.; Gao,

C.; Zhang, L.; Lv, J.; Wang, J.; Zhang, J.; Khattak, A. M.; Khan, N. A.; Wei, Z.;

Zhang, J.; Liu, S.; Zhao, H.; Tang, Z., Ultrathin metal-organic framework nanosheets for electrocatalytic oxygen evolution. Nat. Energy 2016, 1, 16184-16194.

(2) Rui, K.; Zhao, G.; Chen, Y.; Lin, Y.; Zhou, Q.; Chen, J.; Zhu, J.; Sun, W.; Huang, W.; Dou, S. X., Hybrid 2D Dual-Metal-Organic Frameworks for Enhanced Water Oxidation Catalysis. Adv. Funct. Mater. 2018, 28, 1801554.

(3) Hai, G.; Jia, X.; Zhang, K.; Liu, X.; Wu, Z.; Wang, G., High-performance oxygen evolution catalyst using two-dimensional ultrathin metal-organic frameworks nanosheets. Nano Energy 2018, 44, 345-352.

(4) Qian, Q.; Li, Y.; Liu, Y.; Yu, L.; Zhang, G., Ambient Fast Synthesis and Active Sites Deciphering of Hierarchical Foam-Like Trimetal-Organic Framework Nanostructures as a Platform for Highly Efficient Oxygen Evolution Electrocatalysis. 
Adv. Mater. 2019, 31, 1901139-1901147.

(5) Ahn, W.; Park, M. G.; Lee, D. U.; Seo, M. H.; Jiang, G.; Cano, Z. P.; Hassan, F. M.; Chen, Z., Hollow Multivoid Nanocuboids Derived from Ternary Ni-Co-Fe Prussian Blue Analog for Dual-Electrocatalysis of Oxygen and Hydrogen Evolution Reactions. Adv. Funct. Mater. 2018, 28, 1802129.

(6) Xu, Y.; Li, B.; Zheng, S.; Wu, P.; Zhan, J.; Xue, H.; Xu, Q.; Pang, H., Ultrathin two-dimensional cobalt-organic framework nanosheets for high-performance electrocatalytic oxygen evolution. J. Mater. Chem. A 2018, 6, 22070-22076. 